\title{
Internally Generated Error Signals in Monkey Frontal Eye Field during an Inferred Motion Task
}

\author{
Vincent P. Ferrera and Andrei Barborica \\ Departments of Psychiatry and Neuroscience, Columbia University, Keck-Mahoney Center for Brain and Behavior Research, New York, New York 10032
}

An internal model for predictive saccades in frontal cortex was investigated by recording neurons in monkey frontal eye field (FEF) during an inferred motion task. Monkeys were trained to make saccades to the extrapolated position of a small moving target that was rendered temporarily invisible and whose trajectory was altered. On approximately two-thirds of the trials, monkeys made multiple saccades while the target was invisible. Primary saccades were correlated with extrapolated target position. Secondary saccades significantly reduced residual errors resulting from imperfect accuracy of the first saccade. These observations suggest that the second saccade was corrective. Because there was no visual feedback, corrective saccades could only be driven by an internally generated error signal. Neuronal activity in the frontal eye field was directionally tuned before both primary and secondary saccades. Separate subpopulations of cells encoded either saccade direction or direction error before the second saccade. These results suggest that FEF neurons encode the error after the first saccade, as well as the direction of the second saccade. Hence, FEF appears to contribute to detecting and correcting movement errors based on internally generated signals.

\section{Introduction}

Primates use voluntary eye movements to position stimuli onto the foveal region of the retina in which visual acuity is highest. To guide these eye movements, it is useful to be able to anticipate the future location of moving targets (Orban de Xivry et al., 2008) Humans, chimpanzees, and monkeys are able to predict target motion even when the target is rendered temporarily invisible (Filion et al., 1996; Churchland et al., 2003; Bennett and Barnes, 2006; Collier-Baker et al., 2006), although it is unclear whether macaques possess this ability in the same degree as apes (Natale et al., 1986; de Blois and Novak, 1994). The ability to track invisible displacement is considered evidence for the most advanced stage of object permanence (Piaget, 1954) and is impaired in schizophrenia patients (Hooker and Park, 2000).

Moving visual targets may be temporarily occluded and/or change direction. If such changes in visibility and direction of motion are predictable, it is advantageous to move the eyes to a location in which the target is likely to reappear, even while the target remains invisible (Orban de Xivry et al., 2006; Bennett et al., 2007; Mrotek and Soechting, 2007). This location can, in principle, be computed based on an internal model of target motion. Changes in eye position or velocity in response to motor commands are also predictable, and there is evidence that efference copy of commands for both smooth and saccadic eye movements are used in planning predictive movements (Orban de Xivry et al., 2006). An internal model that estimates both future

Received June 10, 2010; accepted July 9, 2010

This work was supported by the Whitehall Foundation and National Institutes of Health Grant MH59244. We thank Franco Pestilli and Tobias Teichert for comments on this manuscript.

Correspondence should be addressed to Dr. Vincent P. Ferrera, Departments of Neuroscience and Psychiatry, Columbia University, 1051 Riverside Drive, Kolb Annex 565, New York, NY 10032. E-mail: vpf3@columbia.edu. DOI:10.1523/JNEUROSCI.2977-10.2010

Copyright $\odot 2010$ the authors $\quad 0270-6474 / 10 / 3011612-12 \$ 15.00 / 0$ target position/velocity and future eye position/velocity can potentially anticipate and correct targeting errors in the absence of visual feedback (de Brouwer et al., 2001, 2002; Blohm et al., 2003, 2005, 2006).

Evidence of an error signal generated in the absence of visual feedback is critical for establishing the existence of an internal model. Figure 1 illustrates a conceptual model of saccade generation that includes a pathway for saccades to invisible targets (represented by elements inside the dashed box). The pathway for invisible targets suggests how various inputs might be combined to produce an error signal. In the model, a memory of target motion is combined with an estimate of elapsed time and other visual cues (i.e., deflector orientation) to produce an estimate of "desired eye position." This is combined with an estimate of actual eye position provided by efference copy of the motor command. The result is an internally generated error signal that feeds into the pathway that transforms signals about visible target motion into saccades.

Previous work has shown that predictive eye movements may be linked to internal representations of invisible target motion in frontal eye field (FEF) (Barborica and Ferrera, 2003, 2004; Xiao et al., 2007). In this study, we report behavioral evidence that monkeys are able to correct targeting errors to invisible moving targets undergoing a change in direction and neurophysiological evidence that an error signal guiding these corrective movements is present in the frontal eye field. The results support the notion that an internal model for eye movement control is present in FEF.

\section{Materials and Methods}

Experiments were performed on three sub-adult male rhesus monkeys (Macaca mulatta). All methods were approved by the Institutional Animal Care and Use Committee at Columbia University and the New York State Psychiatric Institute. Monkeys were prepared for experiments by surgical implantation of a post used for head restraint and a recording 


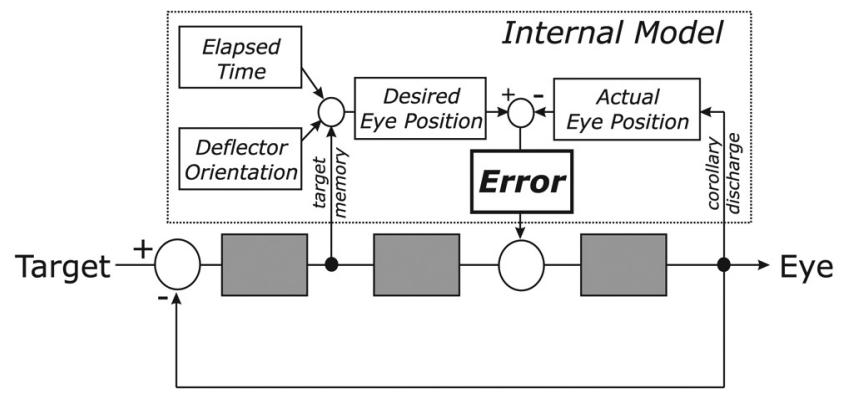

Figure 1. Internal model for saccades to invisible moving targets. The elements within the dashed box represent a pathway for programming saccades to invisible targets. The elements outside the dashed box represent the pathway that generates saccades to visible targets.

chamber to give access to the cortex. Recording chambers were placed at stereotaxic coordinates $20 \mathrm{~mm}$ anterior, $15 \mathrm{~mm}$ lateral (Robinson and Fuchs, 1969). The position of the arcuate sulcus within the recording chamber was visualized through a craniotomy made during the surgery. Eye position was recorded using a monocular scleral search coil (Robinson, 1972; Judge et al., 1980). Monkeys were trained to sit in a primate chair for the duration of the experiment with their heads restrained and to perform visual fixation and tracking tasks for liquid reward.

Visual stimulation. Visual stimuli were generated and controlled by a CRS VSG2/3F video frame buffer. The output from the video board was displayed on a calibrated color monitor with a $60 \mathrm{~Hz}$ non-interlaced refresh rate. The spatial resolution of the display was 1280 pixels $\times 1024$ lines. The central fixation target was a $0.5^{\circ}$ white square, luminance 65 $\mathrm{cd} / \mathrm{m}^{2}$, presented on a uniform dark background. Moving targets were $1.0^{\circ}$ yellow squares, luminance $15 \mathrm{~cd} / \mathrm{m}^{2}$. The frame buffer was programmed to send out digital pulses (frame sync) for timing purposes at the beginning of each video frame in which a target was turned on or off. These pulses were recorded by the computer using a hardware timer and stored together with the eye movement data.

Behavioral tasks. We trained monkeys on an inferred motion task (Fig. 2). The goal was to have monkeys make saccades to a location that could only be inferred from a combination of cues, including the initial velocity of the target, the orientation of a deflector, and the amount of time that the target remained invisible. Monkeys were trained to track a small moving target that became invisible and behaved as if deflected by a visible barrier. At the beginning of each trial, the monkey was required to look at a fixation target in the center of the video display. While the animal was fixating, an oriented bar appeared at the center of the display and a moving target appeared on either the right or left side of the display. The bar and moving target appeared simultaneously. The target moved toward the bar at $10 \% \mathrm{~s}$ for $750 \mathrm{~ms}$. The target then disappeared for a variable amount of time (1250-1750 ms.) When the target reappeared, its direction of motion was changed as if it had been deflected by the bar. The fixation target disappeared $500 \mathrm{~ms}$ before the moving target reappeared and the monkey was allowed to move his eyes in any manner during this period. However, when the target reappeared, the monkey was required to have his gaze direction within $\pm 5^{\circ}$ of the moving target. Although there was technically no fixation requirement during the 500 $\mathrm{ms}$ interval before the reappearance of the moving target, it was advantageous for the monkeys to move their eyes to the location where they expected the target to reappear, and they did this quite reliably. All analyses are based on saccadic eye movements initiated before the reappearance of the moving target.

There were two possible initial target directions; the target either appeared on the left of the screen and moved to the right or appeared on the right and moved to the left. One initial direction was selected randomly on each trial. Bar (deflector) orientation with respect to horizontal was $22.5,45,67,90,112,135$, or $157^{\circ}$. If we define rightward horizontal motion as having a direction of $0^{\circ}$, then a bar that initially started on the left side of the display and moved to the right could be deflected at $45^{\circ}$ (up-right), $90^{\circ}$ (up), $135^{\circ}$ (up-left), $180^{\circ}$ (left), 225 (down-left), $270^{\circ}$ (down), or $315^{\circ}$ (down-right). Targets starting on the right and moving to the left underwent a complementary pattern of deflections. When the deflector was vertical, the target behaved as if it was reflected back toward its initial position; it never behaved as if it passed through the deflector. There were no purely horizontal deflectors.

Occlusion duration was an integral number of video frames randomly selected between 1250 and $1750 \mathrm{~ms}$ (uniform random distribution). This variable interval was used to reduce the likelihood of the monkeys making saccades to a fixed location. The monkey was most likely to be rewarded by making one or more saccades to reach a gaze position appropriate for the target direction and occlusion duration selected for each trial.

Monkeys were also tested on a standard memory-guided saccade task (MEM) with stationary targets. The temporal structure of this task was identical to the motion extrapolation task, with a memory interval (corresponding to the occlusion interval) of variable duration (1250-1750 $\mathrm{ms}$ ). On each trial, the target location was selected from an array of eight positions separated by $45^{\circ}$ around the clock face. Target eccentricity was $10^{\circ}$. Visual feedback was provided by reilluminating the saccade target after the memory-guided saccade was executed. Neural activity was recorded during the memory-guided saccade task to determine the spatial tuning of neurons at the stimulation site.

Neuronal recording. Neural activity was recorded extracellularly using parylene insulated or epoxy-coated tungsten microelectrodes (impedance of $0.15-2.0 \mathrm{M} \Omega$ ). Action potentials were detected using a timeamplitude window and converted to digital pulses with $0.1 \mathrm{~ms}$ precision.

Electrical microstimulation. FEF is defined as the region on the anterior bank of the arcuate sulcus that is active during the initiation of eye movements and from which saccades can be evoked by electrical stimulation (Bruce et al., 1985). Electrical stimulation was therefore used to determine that recording sites were located within FEF. Sites in FEF were located in gray matter on the anterior bank of the arcuate sulcus and were stimulated through the same electrode used to record neuronal activity. The stimulation consisted of a $67 \mathrm{~ms}$ (four video frames) train of biphasic pulses ( $0.2 \mathrm{~ms}$ per phase, cathode leading) at a rate of 350 pulses/s (24 pulses total) and was delivered by an optically isolated pulse stimulator with output compliance up to $100 \mathrm{~V}$ (A-M Systems). The stimulator was gated by a computer-generated digital signal so as to be synchronized with other trial events. The threshold for evoking saccades at each FEF site was determined during a simple fixation task (Opris et al., 2001). During the task, the amplitude of the stimulation current was adjusted to be near or just above the threshold for evoking saccades (pulse amplitude range, $10-100 \mu \mathrm{A}$; one-half peak-to-peak).

Eye movement recording and analysis. Eye position was monitored using a monocular scleral search coil system (CNC Engineering). Separate horizontal and vertical eye position signals were fed through an analog differentiator (low pass, $-3 \mathrm{~dB}$ at $25 \mathrm{~Hz}$ ) to yield horizontal and vertical eye velocity. The eye position and eye velocity signals were then digitally sampled by computer at $1 \mathrm{kHz} / \mathrm{channel}$ and stored on disk for offline analysis. Eye position and velocity records were used to estimate saccade parameters. Radial eye velocity was constructed by combining the magnitudes of the horizontal and vertical eye velocity. Radial eye velocity was digitally differentiated to yield radial eye acceleration. For saccades, movement onset was computed using an acceleration criterion (radial acceleration, $\geq 500^{\circ} / \mathrm{s}^{2}$ ). Saccade offset was found using the complementary criterion (radial acceleration, $\leq 500 \% \mathrm{~s}^{2}$ ). This criterion was not always appropriate for small saccades, hence each trial was also checked by visual inspection. Initial and final eye positions were obtained by taking the average eye position in a $10 \mathrm{~ms}$ window before the beginning or after the end of the saccade, respectively. Electrically evoked saccades were included in the analysis only if they were $>1.5^{\circ}$ in amplitude.

Based on the current eye position and saccadic movements, we defined the following parameters and error signals: saccade direction (SD), which is the direction of the vector defined by the start and end of the saccade; and direction error (DE), which is the direction of the vector linking the eye position at the time of saccade onset with the extrapolated target location at the time of the saccade onset (retrospective DE) or saccade end (prospective DE), respectively.

Smoothing of neuronal tuning functions. Neuronal tuning curves express the dependence of mean firing rate (within a temporal window) on 
some independent variable, e.g., saccade direction or direction error. Estimating smooth tuning functions from noisy firing rates is a challenge, particularly when the temporal window is brief and the independent variable is sampled irregularly. Both of these conditions were essential and unavoidable features of the present experiments. To smooth the tuning curves, we performed a weighted convolution with a symmetric kernel (Gaussian, $\sigma=40^{\circ}$ ) in three steps. The first step was to replace each data point $\left(x_{i}\right.$, $y_{i}$ ) by a Gaussian function of $x$, centered at $x_{i}$, with height proportional to $y_{i}$. This set of Gaussians $\left[G_{i}(x)\right]$ was then summed to yield an initial estimate of the tuning curve $\left[Y_{\text {est }}(x)\right]$.

To account for possible irregular sampling of the independent variable $(x)$, we computed a smooth function that expressed the sampling density of $x$. This was done by substituting a value of 1.0 for each observation, $y_{i}$ in the original data, and then convolving the result with the same Gaussian kernel as above. This yielded a normalization function $\left[Y_{\text {norm }}(x)\right]$. In step three, the final tuning curve was com-

puted as the ratio of the initial estimate and the normalization function:

$$
Y_{\text {mean }}(x)=Y_{\text {est }}(x) / Y_{\text {norm }}(x) .
$$

Note that the set of Gaussians initially computed provides multiple estimates of $Y$ for each value of $x$. These estimates can be used to compute the variance of $Y\left[Y_{\mathrm{var}}(x)\right]$, from which can be derived the SD or SE.

Estimating preferred direction from neuronal tuning functions. To estimate the preferred direction of a neuron based on its tuning for saccade direction or direction error, we used a "center-of-mass" calculation:

$$
\mathbf{P D}=\frac{\Sigma f_{i} \cdot \mathbf{u}_{i}}{\Sigma f_{i}}
$$

where $\mathbf{P D}$ is preferred direction, $f_{i}$ is the firing rate, and $\mathbf{u}_{i}$ is the unit vector of the target direction on the $i$ th trial.

Partial correlation. Partial correlation tests the association between two variables, $x$ and $y$, factoring out their association with a third variable (or set of variables), $Z$. Partial correlation is related to multiple regression because it is the standard correlation of the residuals resulting from the linear regression of $x$ with $Z$ and $y$ with $Z$. When $Z$ is a single variable, the partial correlation coefficient is the following:

$$
r_{x y \cdot Z}=\frac{\left(r_{x y}-r_{x Z} \cdot r_{y Z}\right)}{\sqrt{\left(1-r_{x Z}^{2}\right)\left(1-r_{y Z}^{2}\right)}}
$$

The significance of the partial correlation coefficient, $r_{x y \cdot Z}$, is determined by a modified $t$ test:

$$
t^{\prime}=\frac{r_{x y \cdot z} \sqrt{(n-k-2)}}{\sqrt{1-r_{x y}^{2} \cdot z}}
$$

with $\mathrm{df}=(n-k-2)$. In the present experiments, $k=1$.

Vector reconstruction. The activity of a population of neurons can be used to estimate parameters of the stimulus or eye movement by reducing the activity to a single vector (Georgopoulos et al., 1986; Salinas and Abbott, 1994). To implement this, the tuning function of each neuron was first reduced to a single tuning vector using the center-of-mass equation (Eq. 2). Each tuning vector was normalized (divided by its magnitude) to convert it to a unit vector without changing its direction. To construct a population vector for a particular trial condition (e.g., a particular time epoch within a single trial type), the average firing rate for that condition was computed for each neuron. The unit vector for each cell was then scaled by multiplying it by the average firing rate. The scaled vectors were then summed across all neurons in the population, resulting in a single population vector that represented an estimate of the stimulus or movement direction for that condition.

To incorporate effects of initial target direction, the data for each cell were split into trials in which the target was initially on the right or left side of the display. The split data were used to compute separate two tuning functions and tuning vectors for each neuron. The construction of population vectors for each trial condition was the same as above but performed separately for each initial target direction.

One can construct metrics that express the similarity between the computed population vectors and vectors that represent ideal outcomes. For example, one can compute the similarity between the population vector for a single trial condition with the actual target direction for that condition. One such metric can be computed by taking the inner product of each population vector with the ideal vector for the corresponding trial type and then summing over all trial conditions. This method was used to compute an initial position index expressing the similarity between the population vectors the initial target position and a final position index expressing the similarity between the population vectors and the final target direction.

The directional accuracy of the population vectors relative to target direction was quantified by measuring the angular difference between each population vector and the corresponding vector for either initial or final target position. This yields a reconstruction error that quantifies the fidelity with which the population vectors represent target direction.

\section{Results}

\section{Behavioral analysis of voluntary saccades initiated during the occlusion interval}

To gain insight into the strategy monkeys used to perform the occlusion/deflection task, we analyzed voluntary saccades initiated while the moving target was invisible. Behavioral data were collected during 155 recording sessions ( 76 for monkey A, 21 for monkey C, 58 for monkey D). For these sessions, the final target direction varied among eight possible directions, and the occlusion interval varied from 1250 to $1750 \mathrm{~ms}$ (uniformly distributed). Eye movements made during the occlusion interval could include multiple saccades as well as smooth movements. The present analysis was confined to saccades with minimum amplitude of $1^{\circ}$. The behavioral dataset comprised 25,537 trials, of which 16,473 (64.5\%) contained multiple saccades. 

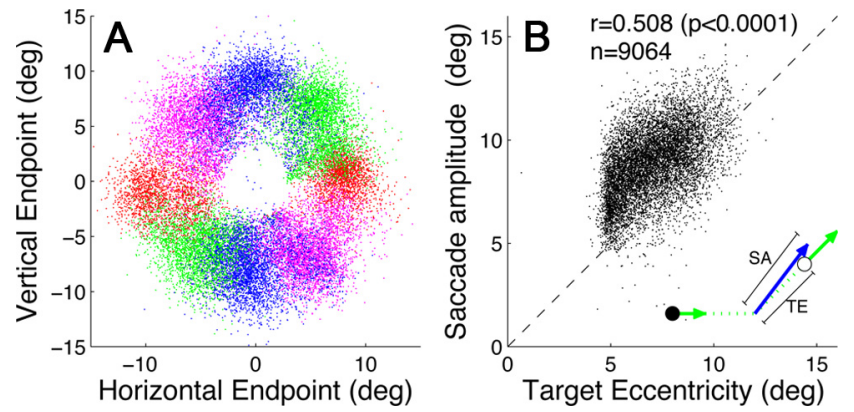

Figure 3. Behavioral analysis of saccades to invisible moving targets for trials with one saccade. $\boldsymbol{A}$, Saccade endpoints color coded by target direction. $\boldsymbol{B}$, Saccade amplitude versus extrapolated target position. $r$ is standard correlation coefficient, $p$ is significance level of the correlation, and $n$ is the number of saccades. The inset diagram illustrates the definitions of extrapolated target position and saccade amplitude. The filled black circle is the initial position of the target. The dotted green line is its trajectory, and the green arrows indicates its initial and final direction of motion. The open circle is the target position at the end of the first saccade. TE is the extrapolated target position relative to the initial fixation position and indicated by the black scale bar. The blue arrow is the saccade. SE and the black scale bar indicate the distance from initial fixation to saccade endpoint.

To determine the degree to which saccades might be guided by an internal representation of the target, we explored the relationship between saccade direction and amplitude and extrapolated target position. Figure $3 A$ shows saccade endpoints sorted by target direction for trials in which a single saccade was initiated during the occlusion interval. The position error of the saccade, defined as the Euclidean distance between the saccade endpoint and the target position extrapolated to the time when the saccade ended, averaged $2.81 \pm 1.27^{\circ}$ (mean $\pm \mathrm{SD}$ ).

The time interval during which the target was invisible before the "go" signal (extinction of the fixation point) was variable, and hence the extrapolated target position also varied. If saccades were driven by an internal representation of target motion, then saccade amplitude should have been correlated with invisible target position at the time of saccade initiation. Figure $3 B$ shows this relationship for trials that had a single saccade. Saccades tended to overshoot the target. Nevertheless, saccade amplitude was significantly correlated with extrapolated target position (Pearson's $r=0.508, p \ll 0.0001, n=9064$ ) (Fig. $3 B$ ).

If saccades initiated during the occlusion interval are driven by an internal representation of target position and/or velocity, then this representation could give rise to an error signal that represents the difference between target position and saccade endpoint. To look for evidence for or against such an error signal, we analyzed trials in which more than one saccade was initiated while the target was invisible. Figure 4 shows the same analysis as Figure $3 B$ but for multiple saccade trials. The amplitude of initial saccades (Fig. $4 a$ ) tended to be weakly correlated with extrapolated target position $(r=0.364, p \ll 0.0001, n=16,473)$. Initial saccades included a population of small-amplitude $\left(<3.0^{\circ}\right)$ saccades that comprised $10 \%$ of the total number of saccades. The small saccades were weakly but significantly correlated with extrapolated target position $(r=0.144, p<0.0001, n=1482)$. They had a mean latency of $190 \mathrm{~ms}$ compared with $210 \mathrm{~ms}$ for the larger saccades $(p<0.0001)$.

The secondary saccades (Fig. $4 b$ ) were significantly better correlated with extrapolated target position $(r=0.543, p \ll 0.0001$, $n=12,084$ ) than first saccades, suggesting that the error after the first saccade was corrected by the second saccade. (There are fewer trials in Figure $4 b$ than in Figure $4 a$ because second saccades
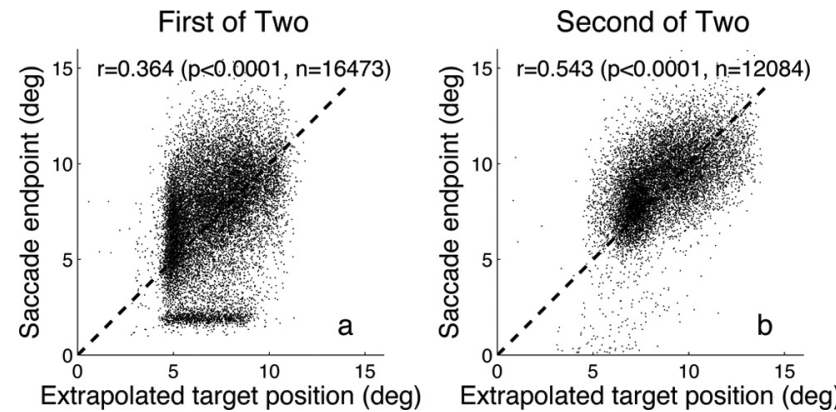

Figure 4. Behavioral analysis of saccades for trials with multiple saccades initiated before the target reappeared. $\boldsymbol{a}$, Saccade amplitude versus extrapolated target position for the first saccade. $r$ is standard correlation coefficient, $p$ is significance level of the correlation, and $n$ is the number of saccades. $\boldsymbol{b}$, Saccade endpoint versus extrapolated target position for the second saccade. Same conventions as $\boldsymbol{a}$.

that were initiated after the reappearance of the target were excluded from the analysis.)

\section{Analysis of position errors}

To further investigate whether the secondary saccades tended to correct the error of the first saccade, we examined the magnitude and direction of the error after the first saccade. Position error is defined as the distance between saccade endpoint and extrapolated target position. Supplemental Figure S1 (available at www. jneurosci.org as supplemental material) shows the distributions of position errors for trials with one saccade (black), the first of two saccades (blue), and the second of two saccades (red). The mean $\pm \mathrm{SD}$ total error (supplemental Fig. S1a, available at www. jneurosci.org as supplemental material) for the second saccade $\left(2.46 \pm 1.34^{\circ}\right)$ was significantly smaller than the mean position errors for either single saccade trials $\left(2.81 \pm 1.27^{\circ}\right.$; $t$ test, $p \ll$ $0.0001)$ or the first of two saccades $\left(3.24 \pm 1.79^{\circ}\right.$; $t$ test, $p \ll$ $0.0001)$. On trials with two saccades, the second saccade reduced position error by an average of $0.78^{\circ}(24 \%)$. The second saccade tended to reduce the position error, although there was no visual feedback.

The total error can be divided into radial and tangential components. The pattern of radial errors (supplemental Fig. S1b, available at www.jneurosci.org as supplemental material) confirms that single saccades tended to overshoot the extrapolated target position (mean radial error is negative), whereas both saccades on multiple saccade trials were more accurate. Tangential error (supplemental Fig. S1c, available at www.jneurosci.org as supplemental material) reflects the direction of the saccade relative to target direction. The bimodality of the distribution for the first of two saccades (blue curve) is attributable to an upward bias that results in a positive direction error for targets moving at $45^{\circ}$ and negative direction error for targets moving at $135^{\circ}$. These errors are corrected by the second saccade.

It seems likely that the monkeys might anticipate where the target was likely to reappear. However, this location was not fixed but varied from trial to trial. Hence, monkeys would still have had to extrapolate the target position based on its speed and elapsed time. This is true whether the saccade goal was the target location at the time of the saccade (for simplicity, call this Ts) or the target location at the time of its reappearance (call this $\mathrm{Tr}$ ). In fact, there was as much trial-to trial-variability in $\mathrm{Tr}$ as in Ts.

We calculated the position error and correlation between saccade endpoint and target location using both $\operatorname{Tr}$ and Ts. We found that, on trials with one saccade, the saccade endpoint was 
equally well correlated with $\operatorname{Ts}(r=0.508)$ or $\operatorname{Tr}(r=0.501)$. However, the total position error was significantly smaller when expressed relative to $\operatorname{Tr}$ (mean error of $1.3^{\circ}$ ) versus Ts (mean error of $2.81^{\circ}$, $t$ test, $\left.p<0.0001\right)$. On trials with multiple saccades, the first saccade was significantly better correlated with Ts $(r=$ $0.369)$ than $\operatorname{Tr}(r=0.305 ; p<0.0001)$. The second saccade was also better correlated with $\operatorname{Ts}(r=0.543)$ than $\operatorname{Tr}(r=0.478)$. However, correlation measures the strength of association, not accuracy. For first and second saccades on multiple saccade trials, the error was significantly smaller relative to $\operatorname{Tr}\left(2.6\right.$ and $1.4^{\circ}$, for first and second saccades, respectively, than relative to Ts $(3.24$ and 2.46, respectively, $t$ test $p<0.001$ ).

This evidence that monkeys plan saccades to the extrapolated target location at the time of reappearance $(\mathrm{Tr})$ is consistent with the idea that they are using a prospective error signal (i.e., they plan saccades to the future location of the target), because the target could be expected to reappear some $2-3^{\circ}$ ahead of its position at the time of the saccade.

\section{Analysis of direction errors}

If the second saccade is corrective, its direction should be correlated with the direction error after the first saccade. Direction error is defined as the direction of the vector defined by the saccade endpoint and target position. There are at least two ways to compute direction error. One is to use the difference between the first saccade endpoint and the target position at the end of the first saccade. This is a retrospective error because it is based on target position before the initiation of the second saccade. The other way to compute direction error is to use the target position at the end of the second saccade. This is a prospective error because it is based on target position after the initiation of the second saccade. Supplemental Figure S2a (available at www. jneurosci.org as supplemental material) shows that there is a significant correlation (circular association) between direction error after the first saccade (retrospective error) and the direction of the second saccade. Note that, when plotted in Cartesian coordinates, saccade direction and direction error are correlated perforce as a result of the fact that the maximum angular difference is $180^{\circ}$. For this reason, the correlations reported are based on circular statistics.

One might expect that second saccades would be correlated with direction error simply because they tended to be directed radially outward. However, this was not necessarily the case. On $12 \%$ of trials with multiple saccades, the first saccade overshot the target and the second saccade was directed radially inward (back toward the center of the display). On these trials, the second saccade was also strongly correlated with direction error $(r=$ 0.851, $n=1478, p \ll 0.0001)$. In fact, the association between saccade direction and direction error for inward saccades was significantly stronger than the comparable correlation for outward saccades $(r=0.716, n=10,606, p \ll 0.0001$.

Figure S2b (available at www.jneurosci.org as supplemental material) uses the prospective direction error and shows that the direction of the second saccade was more closely associated with direction error based not on where the target was at the end of the first saccade but where the target would be at the end of the second saccade. Hence, the secondary saccades compensate for the additional displacement of the target between the end of the first and second saccades.

Secondary saccade direction was also correlated with first saccade direction $(r=0.4, p<0.0001, n=12,084)$ and target direction $(r=0.66, p<0.0001, n=12,084)$. However, neither of these correlations was as strong as the correlation of second sac- cade direction with prospective direction error (supplemental Fig. S2 $b$, available at www.jneurosci.org as supplemental material) $(r=0.872, p<0.0001, n=12,084)$. Thus, prospective direction error explains significantly more of the variance in second saccade direction than any other explanatory variable tested.

\section{Saccade latency}

We looked at whether saccade onsets were clustered around the time of the go signal, or the time of target reappearance. We also investigated the relationship between saccade amplitude and onset time. Supplemental Figure S3 (available at www.jneurosci.org as supplemental material) shows the relationship between saccade amplitude and onset time for single and multiple saccade trials. The onset time of first saccades was typically $\sim 200 \mathrm{~ms}$ after the go signal. First saccade amplitude was not consistently correlated with saccade onset time. Second saccades tended to have a negative correlation between amplitude and onset time. The time of target reappearance was fixed at $500 \mathrm{~ms}$ after the go signal. First saccade onset times are not clustered around the time of target reappearance. Second saccade onsets may be clustered around the time of target reappearance, although this could be confounded with the fact that they tend to occur later in the movement epoch.

\section{Learning effects}

Given the complicated nature of the task, specifically the fact that monkeys needed to integrate three different cues (bar orientation, initial target velocity, and elapsed time), one might expect there to be a learning curve. Unfortunately, we did not record behavioral data while monkeys were initially trained on the task. However, we were able to examine their performance across recording sessions. We looked at the error for first and second saccades as a function of session number. Error generally decreased slightly over sessions, however, none of the trends was significant $(p>0.05)$. Success rate was generally high because the fixation window was large relative to average saccade amplitude $\left( \pm 5^{\circ}\right)$ and was only imposed after the target reappeared. Fluctuations in success rate are difficult to interpret because they could be attributed to motivational factors.

\section{Neuronal activity in FEF during the occlusion interval}

We recorded the activity of 192 FEF neurons in three monkeys. For present purposes, we consider presaccadic activity during time epochs $150 \mathrm{~ms}$ before saccade initiation for saccades made during the target occlusion interval. FEF neurons tend to be narrowly tuned for saccade direction but broadly tuned for saccade amplitude. In our experiment, saccade direction was sampled over a $360^{\circ}$ range, but amplitude was sampled over only a $10-15^{\circ}$ range. Hence, we do not expect many cells to show significant tuning for saccade amplitude and focus instead on tuning for saccade direction.

Figure 5 shows the activity of a single FEF neuron. A sample trial is shown in Figure $5 A$, with a target approaching the deflector from the right and then being deflected down and to the right. Two saccades (gray dots indicate eye position) were initiated while the target was invisible. Trial events as they unfolded in time are represented in Figure 5B. A raster plot and histogram of neuronal activity are shown for 20 trials of the same type. Figure $5 C$ shows action potential histograms aligned to the onset of the first saccade. The dashed vertical lines demarcate the time window within which activity was averaged. This activity is plotted as a function of final target direction in the center of Figure $5 C$ (an example of 

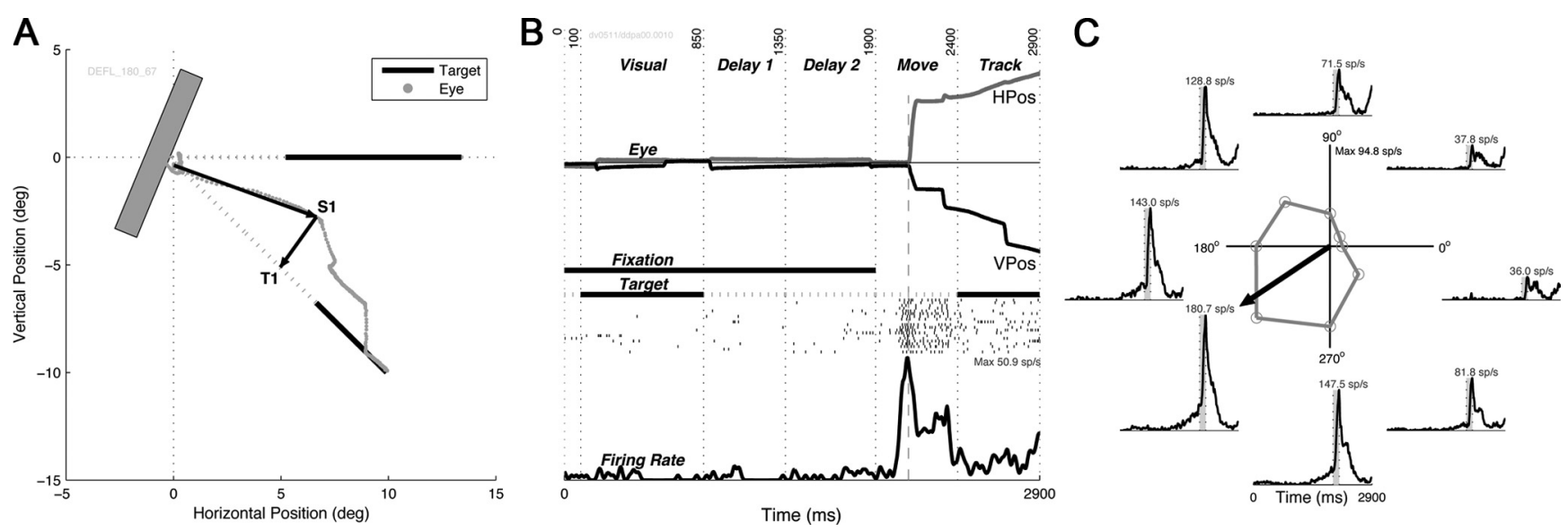

Figure 5. Sample trial, neuronal activity, and tuning curve. $A$, Target trajectory and eye trace for a sample trial having a final target direction of $315^{\circ}$. Dashed segment corresponds to the invisible part of the target trajectory. First saccade vector (S1) and the difference vector between first saccade endpoint and the target position after the first saccade (T1) are shown. $\boldsymbol{B}$, The trial epochs, horizontal (HPos) and vertical (VPos) eye positions, fixation, and target visibility for the trial in $\boldsymbol{A}$ are shown on top of the subplot, and the spike rasters and perisaccade time histograms for all the trials of the same type as the sample trial in $\boldsymbol{A}$ are shown to the bottom. Histograms have been smoothed using a Gaussian kernel having $\sigma=10 \mathrm{~ms}$. $\boldsymbol{C}$, Multigram showing perisaccade time histograms for all eight different target directions in a recording and the tuning curve for the $150 \mathrm{~ms}$ presaccadic activity is shown in the center. The presaccadic interval is marked by the gray rectangle and dashed lines on each perisaccade time histograms. The black arrow points to the preferred direction of the tuning function.

tuning across trial epochs is shown in supplemental Fig. S4, available at www.jneurosci.org as supplemental material).

FEF neurons that have directionally tuned activity before saccade initiation (Fig. 5C) may encode the SD, as defined by its start and end point. Alternatively, they may encode DE, defined by the starting point of the saccade and the position of the target at the start of the saccade. Normally, saccade direction is highly correlated with direction error. This relationship is shown in Figure $6 \mathrm{~A}$ for the first saccade made on each trial during a single recording session. Figure $6 B$ shows the relationship between saccade direction and direction error for the second saccade on trials with multiple saccades. There is still a strong correlation, but it is significantly weaker than the correlation for first saccades $(p \ll$ 0.0001). The somewhat imprecise association between saccade direction and direction error for secondary saccades means that it may be possible to tease apart which of these variables better accounts for the tuning of presaccadic neuronal activity.

The tuning of one FEF neuron was assessed during the same recording session by examining firing rate within an interval 150 $\mathrm{ms}$ before each saccade. Figure $6 \mathrm{C}$ shows the tuning for activity before first (black) and second (red) saccades plotted as a function of retrospective direction error. To generate smooth curves, the firing rate was plotted against direction error for each trial, and then the data were interpolated with a Gaussian function (SD of $40^{\circ}$; see Materials and Methods, Eq. 1). The smoothing procedure compensates for the irregular sampling of direction error. Figure $6 C$ shows that, although the peak presaccadic firing rates for first and second saccades were comparable in magnitude, the tuning curves match rather poorly; the correlation between the first and second saccade tuning functions was not significant $(p>0.5)$.

When the same data were plotted as a function of saccade direction (Fig. 6D), the tuning functions for first (black) and second (blue) saccades matched almost exactly (note that the tuning functions for first saccades are similar whether plotted in terms of direction error, as in Fig. 6C, or saccade direction, as in Fig. 6D). This cell could be referred as a "saccade direction" cell because saccade direction provides a consistent account of its tuning for first and second saccades, although direction error does not.

Other cells had a different pattern of activity. Figure 7 shows another FEF cell whose presaccadic activity was plotted as in
Figure 6. For this cell, direction error (retrospective) provided a consistent account of activity for first and second saccades. In contrast, the tuning curves for saccade direction are not consistent with each other either in shape or the location of their peaks. Hence, this could be referred to as a "direction error" cell. Retrospective and prospective direction errors produced similar tuning functions. However, it was generally easier to distinguish between direction error and saccade direction tuning if prospective direction error was used (see partial correlation analysis below).

To further compare tuning for first and second saccades, we estimated the preferred direction for each cell by computing the center of mass of the tuning function (see Materials and Methods, Eq. 2). We then compared the preferred direction of each cell for first and second saccades. Figure $8 \mathrm{~A}$ shows the distribution of these preferred direction differences. The blue curve is the difference in preferred direction for first and second saccades when neural activity was expressed as a function of saccade direction. The function has a strong peak near $0^{\circ}$, indicating that first and second saccade preferred directions tended to be similar.

The black curve in Figure $8 A$ is the distribution of preferred direction differences for first and second saccades when the tuning was expressed in terms of retrospective direction error. This function also peaks near $0^{\circ}$, but the peak is broader and there are secondary peaks, suggesting that the tuning functions for first and second saccades were less strongly correlated when expressed in terms of direction error. The red curve is the distribution of direction differences when second saccade tuning was expressed in terms of prospective direction error. This distribution has a narrower peak, indicating that there was better agreement between first and second saccade tuning when the latter was expressed in terms of prospective direction error.

One potential problem with comparing tuning for saccade direction and direction error is that the explanatory variables (SD and DE) are themselves correlated. A standard method for dealing with this situation is to use partial correlation. This method allows one to compute the correlation between two variables while factoring out the degree to which each is correlated with a third variable. Figure $8 B$ shows the distribution of partial correlation coefficients for saccade direction and direction error. The partial correlation for saccade direction is the correlation between the saccade direction tuning functions for first (sd1) and 

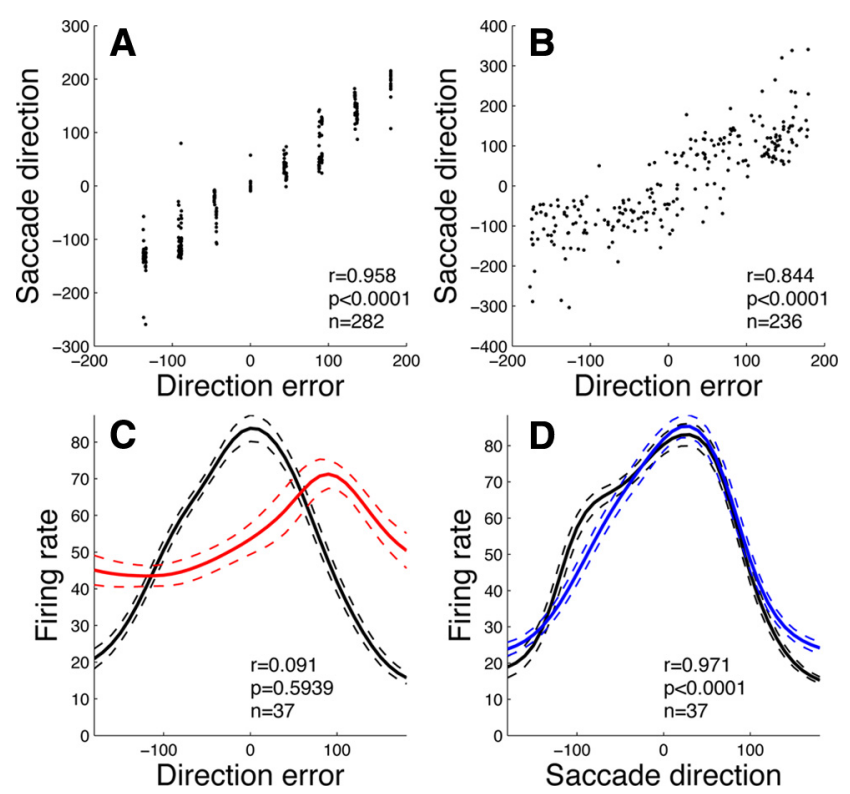

Figure 6. Tuning of an FEF saccade direction neuron. $\boldsymbol{A}$, Dependence of saccade direction on direction error for first saccades. $\boldsymbol{B}$, Saccade direction and direction error for second saccades. $\boldsymbol{C}$, Tuning of FEF presaccadic activity for first (black) and second (red) saccades as a function of direction error. D, Tuning of presaccadic activity for first (black) and second (blue) saccades as a function of saccade direction.

second ( $\mathrm{sd} 2$ ) saccades, with the correlations between saccade direction tuning ( $\mathrm{sd} 1$ and $\mathrm{sd} 2$ ) and direction error tuning for the second saccade $(\mathrm{de} 2)$ partialed out $\left(r_{\mathrm{sd} 1 \mathrm{sd} 2 \cdot \mathrm{de} 2}\right.$; see Materials and Methods, Eq. 3). The partial correlation for direction error is the correlation between the direction error tuning functions for first (de1) and second (de2) saccades, with the correlations between direction error tuning (de1 and de2) and saccade direction tuning $(\mathrm{sd} 2)$ partialed out $\left(r_{\text {delde2 } \cdot \mathrm{sd} 2}\right)$.

The $X$ and + symbols in Figure $8 B$ are cells for which the difference between the saccade direction and direction error partial correlations was significant. In this case, direction error was computed relative to the target position at the end of the first saccade (i.e., retrospective direction error). Seventy-seven cells $(40 \%)$ had a significantly stronger partial correlation for saccade direction than for direction error $(p<0.05)$. Forty-two cells $(22 \%)$ had a significantly stronger partial correlation for direction error.

Behavioral results showed that the direction of secondary saccades was better correlated with prospective direction error (i.e., relative to target position at the end of the second saccade.) Therefore, we also constructed neural tuning curves by plotting firing rate as a function of prospective direction error. The filled symbols in Figure $8 B$ are cells that were significantly $(p<0.05)$ better correlated with saccade direction (blue) or prospective direction error (red.) Using prospective, rather than retrospective, direction error had a small effect on the number of cells significantly correlated with saccade direction $(n=84$ of $192,44 \%$ of total, an increase of 7 cells), but it increased the number of cells significantly tuned for direction error by 57\% $(n=66$ of 192 , $34 \%$ of total, an increase of 24 cells). Thus, direction error cells appear to be more strongly tuned when their activity is expressed in terms of direction error relative to future target position.

The preceding analysis was based on the correlation of the tuning functions for primary and secondary saccades. However, a significant correlation does not guarantee that presaccadic activity is well tuned. To get an appreciation of the shape and strength of the tuning
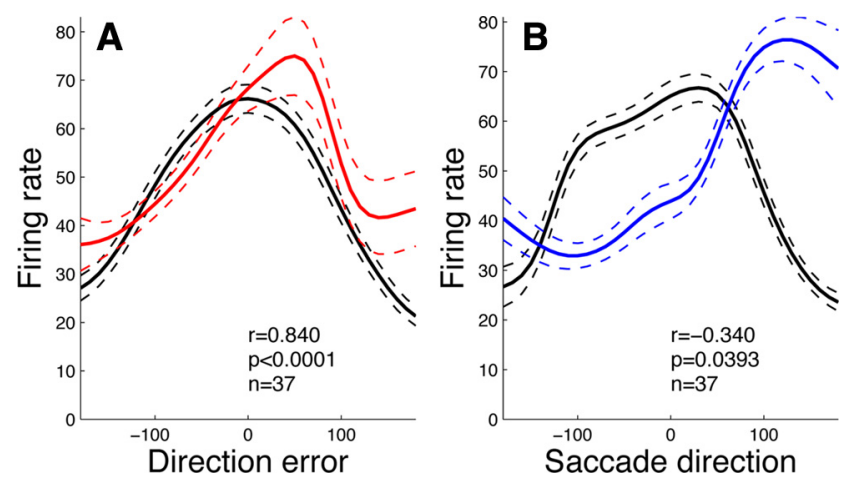

Figure 7. Tuning of an FEF direction error neuron. $\boldsymbol{A}$, Tuning of FEF presaccadic activity for first (black) and second (red) saccades as a function of direction error. $\boldsymbol{B}$, Tuning of presaccadic activity for first (black) and second (blue) saccades as a function of saccade direction.

functions, we constructed population tuning curves for cells that were identified by partial correlation analysis as coding either direction error or saccade direction. The population tuning of direction error cells is plotted in Figure $9 A$ as a function of retrospective direction error ( red curve, $n=42$ cells), prospective direction error (magenta curve, $n=66$ cells), or saccade direction (blue curve, $n=42$ cells). In all cases, "direction" was relative to the preferred direction of tuning for the primary saccade (the tuning function of each cell was rotated so that the preferred directions of all cells lined up at 0 ). The quality of tuning can be evaluated by the following criteria: (1) whether the tuning function was unimodal, (2) whether the peak of the tuning function was close to 0 , and (3) the magnitude of the peak-trough difference. According to these criteria, the tuning functions for direction error cells are well behaved when plotted as a function of retrospective or prospective direction error (although prospective tuning was better than retrospective tuning) but not when plotted as a function of saccade direction. The results for saccade direction cells are complementary (Fig. 9B). Their tuning is better when plotted in terms of saccade direction (blue, $n=77$ cells) than direction error (red, $n=77$ cells). These results independently validate the sorting of cells into direction error and saccade direction classes based on partial correlation.

FEF neurons have been classified as purely visual, purely movement-related or visual-movement (Bruce and Goldberg, 1985). Most neurons in this study were recorded during a memory-saccade task as well as the occlusion/deflection task ( $n=136$ of $192,71 \%$.) The activity during the memory-saccade task can be used to sort cells along a visual-movement continuum. Pure visual cells respond only when the visual cue is presented ( $\mathrm{V}$ is firing rate during cue presentation), whereas pure movement cells respond only before the saccade ( $M$ is firing rate before saccade). A visual-movement index can be computed as follows:

$$
\mathrm{VMI}=(\mathrm{V}-\mathrm{M}) /(\mathrm{V}+\mathrm{M}) .
$$

This ratio varies from 1.0 for purely visual cells to -1.0 for purely movement cells. Of the 136 neurons recorded with both tasks, the average VMI was not significantly different from 0 ( $p=0.35$, range of -1.0 to 1.0$)$. Hence, visual and movement cells were equally represented in the population. In this subset of cells, 107 (56\%) were classified as either saccade direction or direction error cells by the partial correlation analysis. For these cells, there was no significant correlation between VMI and the difference in partial correlation coefficients. 

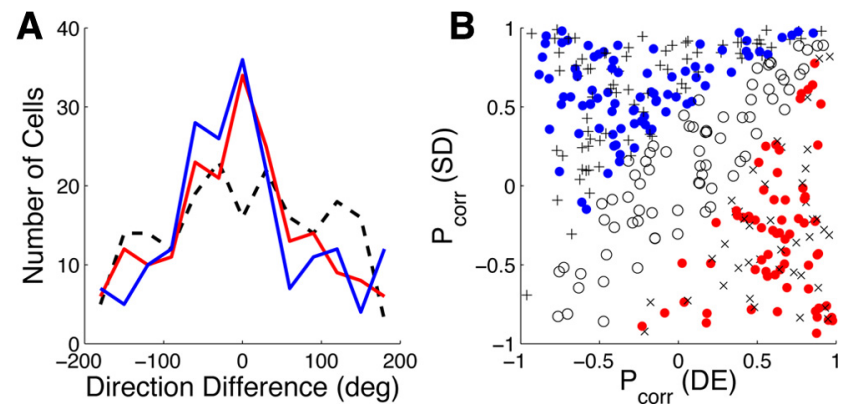

Figure 8. Correlation of FEF tuning functions for first and second saccades. $A$, Distribution of preferred direction differences for first and second saccades when tuning was expressed in terms of saccade direction (blue), direction error relative to target position at the end of the first saccade (black), or direction error relative to target position at the end of the second saccade (red). $\boldsymbol{B}$, Partial correlation for saccade direction and direction error. Open circles are cells with no significant difference. Filled symbols and black + and $\times$ symbols are cells that were significantly ( $p<0.05)$ more strongly correlated with saccade direction ( + and blue circles) or direction error ( $X$ and red circles).

\section{Reconstruction of target trajectory}

To compute an error signal, it is necessary to compare desired and actual eye position. The most likely source for a desired eye position signal is an estimate of extrapolated target position. Because the target trajectory was deflected, the initial and final target directions were different. One can therefore ask whether information about target location (or planned saccade direction) in different task epochs was reflected in FEF activity. Figure $10 a$ shows vectors pointing toward the initial target position. (Target motion vectors would point in the opposite direction as those shown, i.e., blue arrows to the right, red arrows to the left.) This is the pattern that one would expect during decoding activity during the early tasks epochs (visual and early delay) if FEF activity represented the initial target position. Alternatively, it is plausible that FEF neurons encode only final target direction (this may be true even during the visual task epoch, because the moving target and deflector were both visible, allowing the monkey to infer the final target direction). In this case, the decoded pattern of activity would be as shown in Figure $10 b$ (note that we are not distinguishing between final target direction and saccade direction, because these quantities were highly correlated). If FEF neurons encode information about target trajectory, then one should see a transition from the pattern of Figure $10 a$ to that of Figure $10 b$ between the early and late delay epochs. Note that, although we use the phrase "target trajectory," we remain agnostic as to whether FEF activity represents the target, per se, or a plan to acquire the target by making a saccade.

To further clarify, the phrase "information about target trajectory" is not meant to imply "moment-to-moment tracking of target position and velocity." To decode target position, one would need to precisely map the position sensitivity of each neuron during each task epoch. Such data were not acquired in these experiments. Likewise, selectivity for direction of motion was not measured, hence one cannot precisely reconstruct target motion from the present dataset. Given the limitations of the current data, we only sought to determine whether it was possible to decode the direction from the center of the video display (where the monkey's eyes were fixated) to the target location in each trial epoch.

To test whether FEF neurons could encode a rough estimate of target trajectory, i.e., a vector pointing toward the target location in different task epochs, we used standard vector reconstruction methods (see Materials and Methods) to decode the activity of subpopulations of FEF neurons. We first determined that the
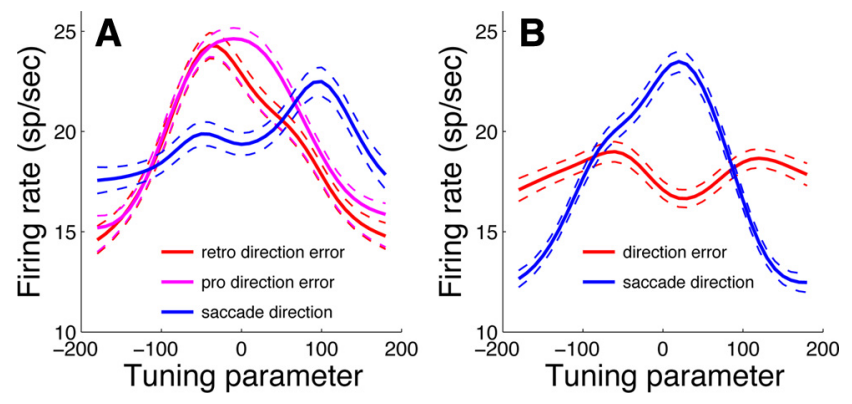

Figure 9. Population tuning of activity preceding secondary saccades. A, Tuning of direction error cells for retrospective direction error (red), prospective direction error (magenta), and saccade direction (blue). $\boldsymbol{B}$, Tuning of saccade direction cells for retrospective direction error (red) and saccade direction (blue). Dashed lines are \pm 1 SEM.

distribution of preferred directions was not significantly different from a circularly uniform distribution (Rayleigh's test, $p=0.38$, Hodges-Ajne test, $p=0.52$ ). We then constructed a population vector for each of the 14 trial conditions ( 2 initial target locations $\times 7$ final target locations), using the activity (mean firing rate) of a subset of cells for each task epoch. We repeated this analysis 1000 times, each time using a randomly selected subset of half the neurons. The resulting population vectors for initial target position during the visual trial epoch (initial time period when the target was visible, before deflection) are plotted in Figure $10 c$ (data for a randomly selected set of iterations are shown as the light red and blue lines, and the red and blue arrows are the vector averages across all iterations). If the visual epoch activity encoded only the initial target position, then all blue vectors should point to the left and all red vectors should point to the right. The distributions in Figure $10 c$ are significantly nonuniform (Rayleigh's test, $p<0.0001$ ) and have horizontal components pointing toward the initial target location, albeit with an upward bias. Note that the initial target motion was in a direction opposite to that of the vectors pointing to the initial target location. Therefore, the population vectors in Figure $10 c$ are not compatible with a representation of the direction of initial target motion.

It is possible that population activity during the visual epoch might also reflect the final target position or saccade plan, given that the monkey had enough information to determine at least the final direction of the target/saccade. Indeed, the target and deflector were both initially visible for $750 \mathrm{~ms}$, allowing the monkey to develop an early plan to initiate saccades to the final extrapolated target location. To test this, the population vectors were sorted by both initial and final target direction and plotted in Figure $10 d$. As one can see, most of the population vectors point in a direction that is intermediate between initial and final target direction. Hence, neuronal activity in FEF reflected information about both the initial target direction and the final target direction even in the earliest task epoch.

We performed the same analysis for activity during the presaccadic epoch before the first saccade. In this case, if neuronal activity encoded the final target direction, the red and blue vectors should overlap. In fact, the population vectors are directed radially outward (Fig. 10e), but the blue vectors generally have a slightly larger net leftward horizontal component than the red vectors, indicating that there is some information about initial target direction even in activity before the first saccade. The same analysis was also performed for activity before the second saccade (Fig. 10f). The results were qualitatively similar to Figure $10 e$. 

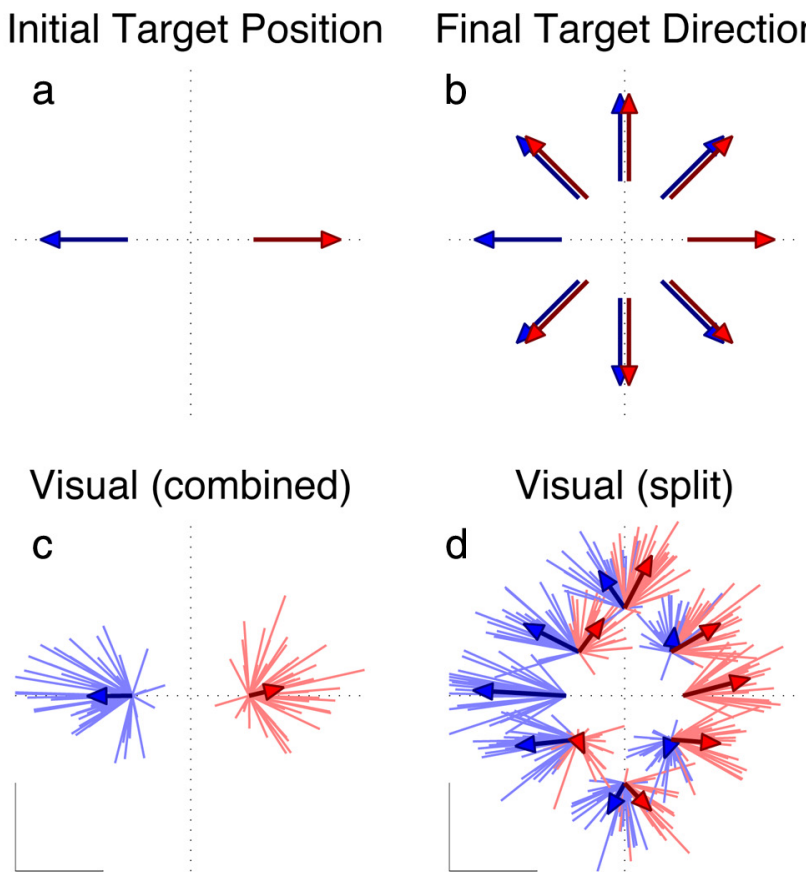

$20 \mathrm{sp} / \mathrm{sec}$

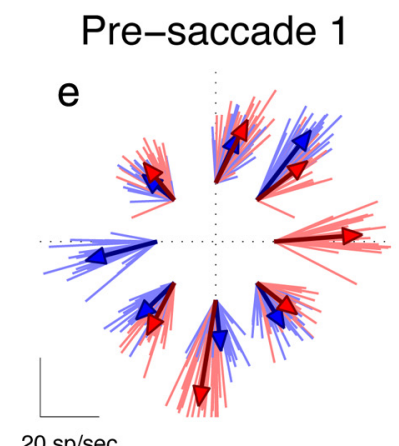

$20 \mathrm{sp} / \mathrm{sec}$

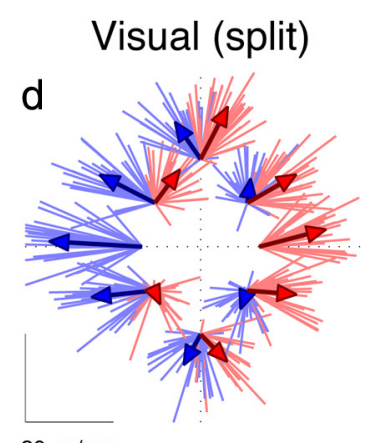

$20 \mathrm{sp} / \mathrm{sec}$

\section{Pre-saccade 2}

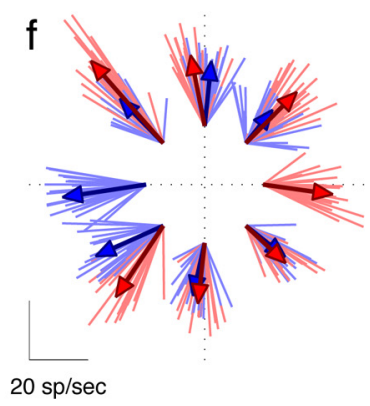

Figure 10. Target trajectory reconstruction. $\boldsymbol{a}$, Vectors pointing toward initial target location for trials in which the target was initially on the right (red) or left (blue) side of the display. $\boldsymbol{b}$, vectors pointing toward the final target direction. $\boldsymbol{c}$, Population vectors during visual trial epoch, combined for all final target directions. $\boldsymbol{d}$, same as $\boldsymbol{c}$, split across different final target directions. Pale red and blue lines were generated using random subpopulations of neurons. Red and blue arrows are averages for each trial condition. $\boldsymbol{e}$, Population vectors during trial epoch $150 \mathrm{~ms}$ before the first saccade. $f$, Population vectors during trial epoch before the second saccade.

One can construct metrics that express the similarity between the population vectors and the hypothetical patterns shown in Figure 10, $a$ and $b$. One such metric simply takes the inner product of each population vector with the predicted vector for the corresponding trial type and sums over trial conditions. This resulted in an initial position index expressing the similarity between the population vectors and the pattern in Figure $10 a$ (initial target position) and a final position index expressing the similarity with the pattern in Figure $10 b$ (final target direction). Both position indices were computed for each trial epoch. The position indices are plotted in Figure 11a. Each point represents the initial and final position indices for a single subpopulation of neurons (again, the analysis was repeated 1000 times on different subpopulations, but only a random fraction of the data is shown).

This analysis establishes several characteristics of the signals available from FEF. First, although there may be individual neurons that are purely visual, there was no subpopulation in the

current sample that exclusively encoded the initial target position, such that the final position index during the visual epoch was equal to 0 (Fig. $11 a$, red points). Every subpopulation of cells contained some information about the final target direction (or, almost equivalently, saccade direction) in every task epoch, including the visual epoch. This was true even if the fraction of cells in the subpopulation was reduced to $25 \%$ of the total and if only the first $150 \mathrm{~ms}$ of the visual epoch was used to estimate firing rate. However, there were subpopulations that were exclusively "movement related" in the sense that they encoded only the final target direction during the presaccadic epochs (initial position index of 0 ) (Fig. 11a, dark blue squares and black dots), although for many subpopulations, presaccadic activity also contained information about the initial target position.

The fidelity with which the population vectors encoded target parameters was quantified by measuring the angular difference between each population vector and the corresponding vector for either initial or final target position. This yields a reconstruction error for each subpopulation of neurons, which was then averaged across subpopulations. The average reconstruction error is shown as a function of trial epoch in Figure $11 b$ (each point is the average of 1000 iterations, and error bars were smaller than the data points). The reconstruction error for final target direction was smaller than that for initial target direction even during the visual task epoch. The fidelity of the estimate of final target direction improved across trial epochs, whereas that for initial target position worsened. Importantly, the reconstruction error for final target direction was reduced by $13 \%$ between the first and second saccades (paired $t$ test, $p<0.0001, n=1000$ ). This could help explain why second saccades tended to be more accurate than first saccades.

One might expect that different cell classes (i.e., those coding saccade direction or direction error) might make different contributions to the accuracy of target direction estimation. Although target direction, saccade direction, and direction error were all significantly correlated, target direction was better correlated with the direction of the second saccade $(r=0.67, n=$ $12,084, p<0.0001)$ than with the direction error after the first saccade $(r=0.33, n=12,084, p<0.0001)$. Hence, one might expect that saccade direction cells would provide a more accurate estimate of target direction than direction error cells. To test this, we selected random subsets of 50 cells drawn from (1) the entire population of cells, (2) only saccade direction cells, or (3) only direction error cells (SD and DE cells were defined using partial correlation, as above, except that the criteria were loosened so that every cell in the population was classified as either DE or SD depending on which partial correlation coefficient was larger). Population vectors were computed for each subpopulation and then used to estimate the target direction reconstruction error based on activity before the first and second saccades, as in Figure $11 b$. This was done with 200 random subpopulations. For subsets drawn from the entire population of cells, the reconstruction error was the same for activity before the first and second saccades (Fig. 11c). For direction error cells, the reconstruction error grew by $12 \%(p<0.05)$ between the first and second saccades. For saccade direction cells, reconstruction error decreased by $35 \%(p<0.001)$. Hence, cell class can have a substantial impact on decoding of target direction.

\section{Discussion}

The internalization of regularities in the environment has the potential to free behavior from dependence on sensory stimuli. Internal representations are useful for movement control because 

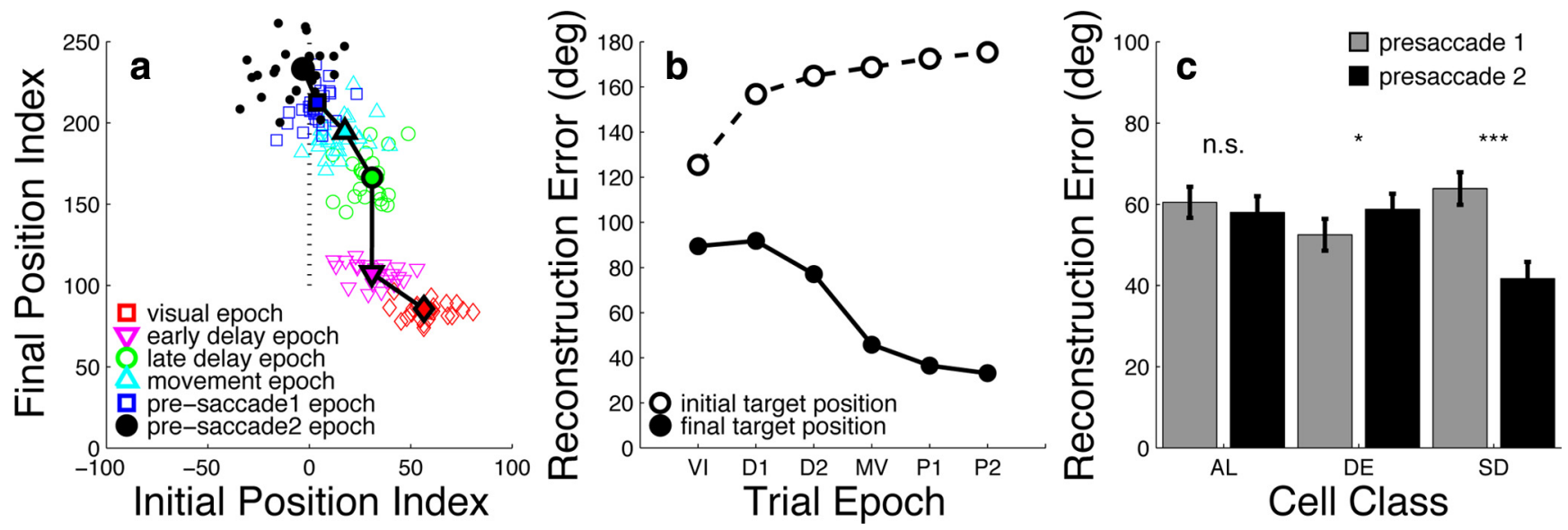

Figure 11. Initial and final target position indices derived from population vectors. $\boldsymbol{a}$, Each small symbol represents the indices computed from a random subpopulation of cells. Large symbols are the mean indices for each trial epoch. $\boldsymbol{b}$, Target position reconstruction error as a function of trial epoch. $\boldsymbol{c}$, Reconstruction error for before first and second saccades using random subsets drawn from all neurons $(A L)$, only direction error neurons (DE), or only saccade direction neurons (SD).

they allow an organism to predict the effects of its own movements relative to internal goals and to plan corrections in the absence of sensory feedback. Without predictive online error monitoring and feedforward correction, complex motor skills, such as playing a musical instrument, might not be possible (Ruiz et al., 2009). Evidence for internally generated error signals is important for establishing the existence of mechanisms that combine internal estimates of the state of the environment and of the effector systems that interact with that environment.

Previous work has established that monkeys can predict the displacement of invisible targets (Filion et al., 1996) and that the frontal eye field may represent the motion of such targets (Barborica and Ferrera, 2003; Xiao et al., 2007) or the motor plan required to intercept them (Barborica and Ferrera, 2004). Monkeys are also capable of compensating for the effects of their own eye movements on the representation of saccade goals, an ability that also involves the frontal eye field (Sommer and Wurtz, 2008). By combining a representation of inferred target motion with signals involved in internal monitoring of eye movements, it should be possible to generate error signals to guide corrective movements in the absence of visual feedback. However, little is know about how this is accomplished.

To gain insight into this process, we trained monkeys to make eye movements toward the location of an invisible moving target whose trajectory was deflected. Monkeys behaved as if they inferred the position of the target based on its initial motion, the orientation of the deflector, and the time elapsed since target disappearance. They frequently initiated multiple saccades while the target was invisible. Primary saccades were correlated with target position, whereas secondary saccades reduced residual position error after the first saccade. The direction of secondary saccades was highly correlated with direction error at the end of the first saccade but was significantly better correlated with direction error relative to future target position (i.e., extrapolated to the end of the second saccade).

These observations suggest that secondary saccades are corrective and are driven by an error signal that is generated in the absence of visual feedback about target position or velocity. The most straightforward way to generate a predictive error signal is to compute the vector difference between the internal representations of target position and eye position to yield a vector that represents the desired saccade. The observation that secondary saccades compensate for the change in extrapolated target posi- tion between the end of the first and second saccade indicates that internally generated error signals also compensate for invisible target velocity, as do saccades to visible targets (Heywood and Churcher, 1981; Ron et al., 1989; Keller and Johnsen, 1990; Eggert et al., 2005; Guan et al., 2005; Cassanello et al., 2008).

Many neurons in the frontal eye field had directionally tuned activity before primary and secondary saccades. This tuning encoded either saccade direction (44\% of neurons) or direction error (34\% of neurons). In parallel to the behavioral results, direction error neurons were better tuned for prospective, rather than retrospective, direction error. Hence, some FEF neurons appear to encode an error signal that compensates for invisible target displacement. The existence of this error signal is evidence in favor of an internal model that combines signals for invisible target motion and eye movement.

The results are consistent with a scenario in which saccades to invisible moving targets are controlled by a continuously varying error signal that evolves over time. The temporal evolution of the error signal (desired eye position) causes primary saccades to be correlated with the extrapolated position of the invisible target. However, after the first saccade, the error is recomputed based on internal estimates of eye position after the first saccade and the future position of the target. This predictive error signal drives a corrective secondary saccade.

To compute an error signal, it is necessary that an estimate of desired eye position be compared with actual eye position. Desired eye position could be derived from an internal representation of target trajectory. We used vector reconstruction methods to determine whether information about target position was present during different task epochs. The reconstruction showed that, during the visual trial epoch, the population of neurons encoded information about both initial and final target location. During later trial epochs, the reconstructed vectors pointed more and more toward the final target location. The reconstructed population vectors suggest that FEF activity could represent either target position as a function of time or an evolving saccade plan. Even during the visual epoch, while the monkey maintained fixation, there could be a covert plan to saccade toward the target.

Temporal evolution of oculomotor signals in frontal cortex has been documented in other paradigms (Kim and Shadlen, 1999; Gold and Shadlen, 2000). However, in those studies, changes in neural activity or motor output over time were attributed to accumulation of sensory evidence rather than inferred 
movement of the saccade goal. Furthermore, the saccade targets were continuously visible so that those studies could not have indicated the presence of internally generated error signals. Nevertheless, time integration appears to be an important mechanism for optimizing behavior and may operate on different types of input depending on whether the task is to make a discrete binary decision or to continuously estimate the trajectory of a moving target. Models that integrate eye velocity to keep track of smooth eye displacement also have been described previously (Blohm et al., 2006).

\section{Relationship to spatial updating}

"Spatial updating" refers to the ability of observers to compensate for their own movements when making saccades to remembered stationary targets. This is typically demonstrated using a "doublestep" saccade paradigm in which two spatially separated targets are briefly illuminated and then extinguished before the onset of eye movement. The subject's task is to make a sequence of two saccades to the remembered locations of the targets. The task is nontrivial because the desired vector of the second saccade does not correspond to the retinal error of the second target but must be computed by taking the difference between the retinal error of the second target and the first saccade vector. Humans and monkeys can perform the double-step saccade task accurately, and a number of studies have documented signals in monkey FEF that are of potential value in performing the internal computation of the second saccade vector (Segraves and Goldberg, 1987; Segraves, 1992; Umeno and Goldberg, 1997; Schiller and Chou, 2000; Tian et al., 2000; Cassanello and Ferrera, 2007; Sommer and Wurtz, 2008).

There are several important differences between previous spatial updating studies and the current experiments. Most previous studies of spatial updating in FEF did not use moving targets. The double-step saccade task requires an internal representation of the position of the saccade goal that is updated by subtracting or undoing the effect of the subject's eye initial eye movement. However, it does not require that the saccade goal be continuously updated based on its inferred movement. The present study presents evidence that saccades compensate for the motion of the invisible target, which requires an internal representation of target velocity as well as position.

Previous studies (Umeno and Goldberg, 1997; Tian et al., 2000) have shown predictive signals in FEF that appear just before saccade initiation. However, they did not investigate the tuning of these signals nor did they test whether these signals represent saccade direction or direction error. The current data show that FEF presaccadic activity is tuned and that the tuning of some cells is better described by saccade direction, whereas other cells are better correlated with direction error. The direction error and saccade direction signals could represent distinct computational stages. One hypothesis is that direction error cells compute an error signal by comparing the outcome of the actual previous movement with the future target location, whereas saccade direction cells read out the activity of direction error cells to generate a representation of the upcoming movement.

A question naturally arises as to why saccade direction is not more closely correlated with direction error. This could be attributable to the noise inherent in these signals and reflected in the variability of movements to remembered or inferred targets. The desired eye position driving both primary and secondary saccades in the occlusion/deflection task does not correspond to any visual stimulus, or even the memory of a visual stimulus, but must be constructed by piecing together several pieces of infor- mation: the initial target motion, the bar orientation, and the elapsed time. It is not surprising that such a signal is inherently noisy. Internal estimates of eye position are also likely to be quite variable. Even an optimal decoder (Jazayeri and Movshon, 2006) will have noisy output when the signal it is trying to read out is represented by broadly tuned neurons that have a high degree of variability. Previous information may also come into play when decoding such signals (Wolpert et al., 1995) and may provide another experimental tool for dissociating direction error and saccade direction.

\section{Conclusion}

We have attempted to provide experimental evidence for internally generated FEF signals that are present when initiating saccadic eye movements and subsequent corrective saccades in the absence of immediate visual stimulation. The existence of an error signal for corrective eye movements is supported by the following main findings. (1) Monkeys make secondary saccades on approximately two-thirds of the trials and in the absence of visual feedback. (2) Secondary saccades reduce position error. (3) Secondary saccade direction is correlated with direction error. (4) Secondary saccade direction is better correlated with prospective than retrospective direction error. (5) FEF neurons have activity that is directionally tuned before both primary and secondary saccades. (6) The tuning of a subpopulation of FEF neurons was significantly better explained by direction error than by saccade direction.

\section{References}

Barborica A, Ferrera VP (2003) Estimating invisible target speed from neuronal activity in monkey frontal eye field. Nat Neurosci 6:66-74.

Barborica A, Ferrera VP (2004) Modification of saccades evoked by electrical stimulation of macaque frontal eye field during invisible target tracking. J Neurosci 24:3260-3267.

Bennett SJ, Barnes GR (2006) Combined smooth and saccadic ocular pursuit during the transient occlusion of a moving visual object. Exp Brain Res 168:313-321.

Bennett SJ, Orban de Xivry JJ, Barnes GR, Lefèvre P (2007) Target acceleration can be extracted and represented within the predictive drive to ocular pursuit. J Neurophysiol 98:1405-1414.

Blohm G, Missal M, Lefèvre P (2003) Interaction between smooth anticipation and saccades during ocular orientation in darkness. J Neurophysiol 89:1423-1433.

Blohm G, Missal M, Lefèvre P (2005) Processing of retinal and extraretinal signals for memory-guided saccades during smooth pursuit. J Neurophysiol 93:1510-1522.

Blohm G, Optican LM, Lefèvre P (2006) A model that integrates eye velocity commands to keep track of smooth eye displacements. J Comput Neurosci 21:51-70.

Bruce CJ, Goldberg ME (1985) Primate frontal eye fields. I. Single neurons discharging before saccades. J Neurophysiol 53:603-635.

Bruce CJ, Goldberg ME, Bushnell MC, Stanton GB (1985) Primate frontal eye field. II. Physiological and anatomical correlates of electrically evoked eye movements. J Neurophysiol 54:714-734.

Cassanello CR, Ferrera VP (2007) Computing vector differences using a gain field-like mechanism in monkey frontal eye field. J Physiol 582: 647-664.

Cassanello CR, Nihalani AT, Ferrera VP (2008) Neuronal responses to moving targets in monkey frontal eye fields. J Neurophysiol 100:1544-1556.

Churchland MM, Chou IH, Lisberger SG (2003) Evidence for object permanence in the smooth-pursuit eye movements of monkeys. J Neurophysiol 90:2205-2218.

Collier-Baker E, Davis JM, Nielsen M, Suddendorf T (2006) Do chimpanzees (Pan troglodytes) understand single invisible displacement? Anim Cogn 9:55-61.

de Blois ST, Novak MA (1994) Object permanence in rhesus monkeys (Macaca mulatta). J Comp Psychol 108:318-327.

de Brouwer S, Missal M, Lefèvre P (2001) Role of retinal slip in the predic- 
tion of target motion during smooth and saccadic pursuit. J Neurophysiol 86:550-558.

de Brouwer S, Yuksel D, Blohm G, Missal M, Lefèvre P (2002) What triggers catch-up saccades during visual tracking? J Neurophysiol 87:1646-1650.

Eggert T, Guan Y, Bayer O, Büttner U (2005) Saccades to moving targets. Ann N Y Acad Sci 1039:149-159.

Filion CM, Washburn DA, Gulledge JP (1996) Can monkeys (Macaca mulatta) represent invisible displacement? J Comp Psychol 110:386-395.

Georgopoulos AP, Schwartz AB, Kettner RE (1986) Neuronal population coding of movement direction. Science 233:1416-1419.

Gold JI, Shadlen MN (2000) Representation of a perceptual decision in developing oculomotor command. Nature 404:390-394.

Guan Y, Eggert T, Bayer O, Büttner U (2005) Saccades to stationary and moving targets differ in the monkey. Exp Brain Res 161:220-232.

Heywood S, Churcher J (1981) Saccades to step-ramp stimuli. Vision Res 21: 479-490.

Hooker C, Park S (2000) Trajectory estimation in schizophrenia. Schizophr Res 45:83-92.

Jazayeri M, Movshon JA (2006) Optimal representation of sensory information by neural populations. Nat Neurosci 9:690-696.

Judge SJ, Richmond BJ, Chu FC (1980) Implantation of magnetic search coils for measurement of eye position: an improved method. Vision Res 20: $535-538$.

Keller E, Johnsen SD (1990) Velocity prediction in corrective saccades during smooth-pursuit eye movements in monkey. Exp Brain Res 80: 525-531.

Kim JN, Shadlen MN (1999) Neural correlates of a decision in the dorsolateral prefrontal cortex of the macaque. Nat Neurosci 2:176-185.

Mrotek LA, Soechting JF (2007) Predicting curvilinear target motion through an occlusion. Exp Brain Res 178:99-114.

Natale F, Antinucci F, Spinozzi G, Poti P (1986) Stage 6 object concept in nonhuman primate cognition: a comparison between gorilla (Gorilla gorilla gorilla) and Japanese macaque (Macaca fuscata). J Comp Psychol 100:335-339.

Opris I, Barborica A, Ferrera VP (2001) On the gap effect for saccades evoked by electrical stimulation of the frontal eye fields in monkeys. Exp Brain Res 138:1-7.
Orban de Xivry JJ, Bennett SJ, Lefèvre P, Barnes GR (2006) Evidence for synergy between saccades and smooth pursuit during transient target disappearance. J Neurophysiol 95:418-427.

Orban de Xivry JJ, Missal M, Lefevre P (2008) A dynamic representation of target motion drives predictive smooth pursuit during target blanking. J Vis $8: 1-13$.

Piaget J (1954) The construction of reality in the child. New York: Norton.

Robinson DA (1972) Eye movements evoked by collicular stimulation in the alert monkey. Vision Res 12:1795-1808.

Robinson DA, Fuchs AF (1969) Eye movements evoked by stimulation of frontal eye fields. J Neurophysiol 32:637-648.

Ron S, Vieville T, Droulez J (1989) Use of target velocity in saccadic programming. Brain Behav Evol 33:85-89.

Ruiz MH, Jabusch HC, Altenmüller E (2009) Detecting wrong notes in advance: neuronal correlates of error monitoring in pianists. Cereb Cortex 19:2625-2639.

Salinas E, Abbott LF (1994) Vector reconstruction from firing rates. J Comput Neurosci 1:89-107.

Schiller PH, Chou I (2000) The effects of anterior arcuate and dorsomedial frontal cortex lesions on visually guided eye movements in the rhesus monkey. 1. Single and sequential targets. Vis Res 40:1609-1626.

Segraves MA (1992) Activity of monkey frontal eye field neurons projecting to oculomotor regions of the pons. J Neurophysiol 68:1967-1985.

Segraves MA, Goldberg ME (1987) Functional properties of corticotectal neurons in the monkey s frontal eye field. J Neurophysiol 58:1387-1419.

Sommer MA, Wurtz RH (2008) Brain circuits for the internal monitoring of movements. Annu Rev Neurosci 31:317-338.

Tian J, Schlag J, Schlag-Rey M (2000) Testing quasi-visual neurons in the monkey's frontal eye field with the triple-step paradigm. Exp Brain Res 130:433-440.

Umeno MM, Goldberg ME (1997) Spatial processing in the monkey frontal eye field. I. Predictive visual responses. J Neurophysiol 78:1373-1383.

Wolpert DM, Ghahramani Z, Jordan MI (1995) An internal model for sensorimotor integration. Science 269:1880-1882.

Xiao Q, Barborica A, Ferrera VP (2007) Modulation of visual responses in macaque frontal eye field during covert tracking of invisible targets. Cereb Cortex 17:918-928. 